\title{
Study of measurement and calculation of the relative amplitude of accommodation
}

\author{
Pilar Coloma, Inmaculada Pascual, Dolores de Fez and Vicente Camps \\ Dept of Optics, Pharmacology and Anatomy, University of Alicante, Spain
}

\begin{abstract}
Status of binocular vision is evaluated using binoculars and accommodative tests. The results of these tests allow the optometrist to decide or the type of lenses has to be prescribed or whether a vision therapy is required.

The aim of this work is to check the accuracy of the measurement of the relative amplitude of accommodation.

Relative accommodation amplitude assesses the ability to increase or decrease the accommodation when convergence demand is constant. The eye must be neutralized to take the measurement. Because the test is situated at a distance of 40 $\mathrm{cm}$, it is considered that the convergence is 2'5 am and the accommodation 2'50 D. However, this assumption could lead to important errors in ammetropic subjects due to the eye after neutralization accommodates in the image produced by the lens forms and converges in the position of the effective binocular object [1]. Therefore, it is necessary to set measurement conditions in which the accommodative and convergence stimulus are the same for all subjects.
\end{abstract}

Furthermore, the lenses added on the neutralization to determine the relative amplitude of accommodation do not correspond to the dioptric value of relaxation and stimulation of the eye accommodation. To obtain this value a more precise calculation has to be performed.

Keywords: accommodation, convergence, emmetrope, ammetrope, neutralization, relative amplitude of accommodation, binocular vision, effective binocular object.

\section{INTRODUCTION}

The relative amplitude of accommodation is usually measured in the binocular subjective examination presenting a near vision test at a distance of $40 \mathrm{~cm}$ from the glasses or the phoropter. Let us denote by $\mathrm{P}_{\mathrm{NL}}$ the power of the neutralizing lens. Positive binocular lenses are added in 0'25D steps until the subject manifests the first maintained blurriness. The positive lenses added to the subjective result are the value of the negative relative accommodation (NRA). After, binocular negative lenses are added in 0'25D steps until the subject manifests the first maintained blurriness. The value of negative lenses added is the value of the positive relative accommodation (NRP). Normal values at a distance of 40 cm: NRA: $+2 ' 00 \mathrm{D} \pm 0.50$ and ARP-2'37 \pm 0 '50D. [2][3][4][5] [6]

Under these conditions it is assumed that the stimulus at $40 \mathrm{~cm}$ produces 2'50 D of accommodation and 2.50 am of convergence. When the eye is emmetrope, without lenses, we could affirm that this statement is true if the distance of 40 $\mathrm{cm}$ is measured from the eye to test. However, in neutralized ammetropia, accommodation and convergence in these conditions is no longer the same.

Keep in mind that normal values used in most binoculars tests are usually determined from emmetropic populations, it is important to know whether they can be used also in ammetropic populations.

A neutralized ammetropic subject does not accommodate in the test position, but at the position where the neutralizing lens forms its image. The neutralized acomodation $\left(\mathrm{A}_{\mathrm{N}}\right)$ depends on the $\mathrm{P}_{\mathrm{NL}}$, as mentioned above [7]:

$$
A_{N}=\frac{X^{\mathrm{Hoc}}\left(1+\delta_{v}^{\mathrm{Hoc}} R^{\mathrm{Hoc}}\right)^{2}}{X^{\mathrm{Hoc}}\left(\delta_{\mathrm{V}}^{\mathrm{Hoc}}\right)^{2} \mathrm{R}^{\mathrm{Hoc}}-1}
$$

8th Iberoamerican Optics Meeting and 11th Latin American Meeting on Optics, Lasers, and Applications,

edited by Manuel Filipe P. C. Martins Costa, Proc. of SPIE Vol. 8785, 878597

(C) 2013 SPIE · CCC code: $0277-786 X / 13 / \$ 18 \cdot$ doi: $10.1117 / 12.2027493$ 
where $\mathrm{X}^{\mathrm{Hoc}}$ is the vergence of the test distance taking as origin of measurement the object principal plane of the eye (Hoc), $\delta_{\mathrm{V}}^{\text {Hoc }}$ is the distance from the object principal plane of the eye to the neutralizing lens and $\mathrm{R}^{\text {Hoc }}$ is the vergence of the remote point measured from the object principal plane of the eye.

As we mentioned above also, a neutralized ammetropic subject does not converge in the plane of the test, but in the position of the effective binocular object. This is because the eye must rotate to maintain foveal fixation when the neutralizing lens is placed in front of eye. [1]

The position of the effective binocular object can be calculated with the following expression [8]:

$$
x^{B}=x-x^{G} \cdot q^{G} \cdot P_{N L}
$$

where: $x^{B}$ is the position of the binocular effective object measured from the corneal vertex, $x$ is the test distance measured from the corneal apex, $\mathrm{x}^{\mathrm{G}}$ is the test distance measured from the lens and $\mathrm{q}^{\mathrm{G}}$ is the distance of the center of rotation of the eye measured from the lens.

To asses how the value of the neutralizing lens affects the values of accommodation and convergence, two different calculations were carried out for different values of neutralizing lens. First, the accommodation performed considering the position of the test at $40 \mathrm{~cm}$ through the neutralizing lens and the convergence considering the position of the effective binocular object position (see Table 1)

Table 1. This table shows the accommodation and convergence values for different $\mathrm{P}_{\mathrm{NL}}$ values.

\begin{tabular}{|l|c|c|c|c|c|c|c|c|c|c|}
\cline { 2 - 11 } \multicolumn{1}{c|}{} & \multicolumn{9}{c|}{$\mathbf{P}_{\text {NL }}$ (D) } \\
\cline { 2 - 11 } \multicolumn{1}{c|}{} & $\mathbf{- 2}$ & $\mathbf{- 4}$ & $\mathbf{- 6}$ & $\mathbf{- 8}$ & $\mathbf{- 1 0}$ & $\mathbf{+ 2}$ & $+\mathbf{4}$ & $+\mathbf{+ 6}$ & $\mathbf{+ 8}$ & $+\mathbf{1 0}$ \\
\hline $\mathbf{A}_{\mathbf{N}}$ (D) & $2^{\prime} 32$ & $2^{\prime} 21$ & $2^{\prime} 12$ & $2^{\prime} 03$ & $1^{\prime} 94$ & $2^{\prime} 55$ & $2^{\prime} 67$ & $2^{\prime} 81$ & $2^{\prime} 96$ & $3^{\prime} 12$ \\
\hline $\mathbf{C}(\mathbf{a m})$ & $2^{\prime} 31$ & $2^{\prime} 21$ & $2^{\prime} 11$ & $2^{\prime} 03$ & $1^{\prime} 95$ & $2^{\prime} 55$ & $2^{\prime} 69$ & $2^{\prime} 85$ & $3^{\prime} 03$ & $3^{\prime} 23$ \\
\hline
\end{tabular}

As it can be seen in the tables above, accommodation and convergence values vary for different values of $\mathrm{P}_{\mathrm{LN}}$. Furthermore, the values of accommodation and convergence are equal for each neutralizing lens, but these are not the same which would have an emmetropic subject with the test located at $40 \mathrm{~cm}$. Therefore, the starting conditions to performing the binocular optometric test when it is located at $40 \mathrm{~cm}$ will not be the same for emmetropic and ammetropic subjects.

\section{ANALYSIS CALCULATION METHOD}

In order to the convergence stimulus is equal to ammetropic and emmetropic subjects, we calculated what should be the position of the test such the effective binocular object position was at $40 \mathrm{~cm}$ from the spectacle plane in ammetropic subjects. In these circumstances, the accommodation values are obtained for different $\mathrm{P}_{\mathrm{LN}}$. The convergence, would be 2.50 am in all cases.

Table 2. Distance $\left(\mathrm{D}^{\mathrm{G}}\right)$ of the test measured from the spectacle planes when the effective binocular object is at $40 \mathrm{~cm}$ of the lens, in miopic and hipermetropic subjects and the corresponding accommodation values.

\begin{tabular}{|c|c|c|c|c|c|c|c|c|c|c|}
\cline { 2 - 12 } \multicolumn{1}{c|}{} & \multicolumn{9}{c|}{$\mathbf{P}_{\mathbf{N L}}(\mathbf{D})$} \\
\cline { 2 - 11 } \multicolumn{1}{c|}{} & $\mathbf{- 2}$ & $\mathbf{- 4}$ & $\mathbf{- 6}$ & $\mathbf{- 8}$ & $\mathbf{- 1 0}$ & $\mathbf{+ 2}$ & $\mathbf{+ 4}$ & $\mathbf{+ 6}$ & $\mathbf{+ 8}$ & $+\mathbf{1 0}$ \\
\hline $\mathbf{x}^{\mathbf{G}} \mathbf{( c m )}$ & $38^{\prime} 1$ & $36^{\prime} 3$ & $34^{\prime} 7$ & $33^{\prime} 2$ & $31^{\prime} 9$ & $42^{\prime} 1$ & $44^{\prime} 5$ & $47^{\prime} 2$ & $50^{\prime} 3$ & $53^{\prime} 7$ \\
\hline $\mathbf{A}_{\mathbf{N}}(\mathbf{D})$ & $2^{\prime} 51$ & $2^{\prime} 51$ & $2^{\prime} 51$ & $2^{\prime} 51$ & $2^{\prime} 51$ & $2^{\prime} 49$ & $2^{\prime} 47$ & $2^{\prime} 45$ & $2^{\prime} 43$ & $2^{\prime} 40$ \\
\hline
\end{tabular}


As can be seen in Table 2, the position of the test is modified, the values of accommodation and convergence in all cases are practically the same and equal those obtained by an emmetropic subject if thethe test is at $40 \mathrm{~cm}$. There are small variations in the value of the amplitude of accommodation in hyperopia, although these do not exceed 0 '25D and are not clinically relevant. For calculation has been obtained $x^{G}$ value of equation. (2).

As we have commented above, to consider that the lenses added have the same dioptric value that the variation produced in accommodation will lead to an error. To quantify this error, we calculated theoretically the value of the NRA and NRA for different values of $\mathrm{P}_{\mathrm{NL}}$ and different values of added lenses, both positive and negative. The calculation was performed as follows:

1. Calculation of the position of the test image through the $\mathrm{P}_{\mathrm{NL}}$ (called $\mathrm{X}^{\prime}$ ), using the Gauss equation:

$$
\mathrm{X}^{\prime}=\mathrm{X}+\mathrm{P}_{\mathrm{LN}}
$$

where $\mathrm{X}^{\prime}$ is the vergence of the image and $\mathrm{X}$ is the vergence of the object.

2. Calculation of the image of the test through added lenses, positive $(\mathrm{L}+)$ or negative (L-), if NRA or PRA are measured respectively, using the Gauss equation. The vergence image has been called X'.

3. Calculation of accommodation performed by the eye, depending on the value of the refraction at the position $\mathrm{X}^{\prime}$ :

$$
\mathrm{A}=\mathrm{R}-\mathrm{X}^{\prime}
$$

4. Calculation of accommodation performed by the eye, depending on their refraction value, in the position of X", with equation (3):

$$
\mathrm{A}=\mathrm{R}-\mathrm{X}^{\prime \prime}
$$

5. Difference between the value of accommodation at $X^{\prime \prime}$ and at $X^{\prime}$. This difference would indicate the variation in accomodation when the lenses are added, and therefore this difference will provide the relative accommodation PRA and NRA.

The results of the calculations are shown below in Table 3 (NRA) and Table 4 (PRA). 
Table 3. NRA theoretical values based on the positive lenses added and the value of the myopic and hyperopic $\mathrm{P}_{\mathrm{NL}}$.

\begin{tabular}{|l|l|l|l|l|l|l|l|l|l|l|}
\cline { 2 - 12 } \multicolumn{1}{c|}{} & \multicolumn{9}{|c|}{$\mathbf{P}_{\mathbf{N L}}(\mathbf{D})$} \\
\hline $\mathbf{L}(+\mathbf{)}$ & $\mathbf{- 2}$ & $\mathbf{- 4}$ & $\mathbf{- 6}$ & $\mathbf{- 8}$ & $\mathbf{- 1 0}$ & $\mathbf{+ 2}$ & $\mathbf{+ 4}$ & $\mathbf{+ 6}$ & $\mathbf{+ 8}$ & $+\mathbf{1 0}$ \\
\hline $\mathbf{+ 0 , 2 5}$ & 0,22 & 0,21 & 0,20 & 0,19 & 0,18 & 0,25 & 0,26 & 0,28 & 0,29 & 0,31 \\
\hline $\mathbf{+ 0 , 5}$ & 0,45 & 0,42 & 0,40 & 0,39 & 0,37 & 0,50 & 0,52 & 0,56 & 0,59 & 0,62 \\
\hline $\mathbf{+ 0 , 7 5}$ & 0,67 & 0,64 & 0,61 & 0,58 & 0,55 & 0,75 & 0,79 & 0,84 & 0,89 & 0,94 \\
\hline$+\mathbf{1}$ & 0,90 & 0,85 & 0,81 & 0,78 & 0,74 & 1,00 & 1,06 & 1,12 & 1,19 & 1,26 \\
\hline$+\mathbf{1 , 2 5}$ & 1,13 & 1,07 & 1,02 & 0,97 & 0,93 & 1,25 & 1,33 & 1,40 & 1,49 & 1,58 \\
\hline$+\mathbf{1 , 5}$ & 1,36 & 1,29 & 1,23 & 1,17 & 1,12 & 1,51 & 1,60 & 1,69 & 1,79 & 1,90 \\
\hline$+\mathbf{1 , 7 5}$ & 1,59 & 1,51 & 1,44 & 1,37 & 1,31 & 1,77 & 1,87 & 1,98 & 2,10 & 2,23 \\
\hline$+\mathbf{2}$ & 1,82 & 1,73 & 1,65 & 1,57 & 1,50 & 2,03 & 2,14 & 2,27 & 2,41 & 2,56 \\
\hline$+\mathbf{2 , 2 5}$ & 2,06 & 1,95 & 1,86 & 1,77 & 1,69 & 2,29 & 2,42 & 2,56 & 2,72 & 2,89 \\
\hline$+\mathbf{2 , 5}$ & 2,29 & 2,18 & 2,07 & 1,97 & 1,88 & 2,55 & 2,70 & 2,86 & 3,03 & 3,22 \\
\hline $\mathbf{2 , 7 5}$ & 2,53 & 2,40 & 2,29 & 2,18 & 2,08 & 2,82 & 2,98 & 3,16 & 3,35 & 3,56 \\
\hline$+\mathbf{3}$ & 2,77 & 2,63 & 2,50 & 2,38 & 2,27 & 3,08 & 3,26 & 3,46 & 3,67 & 3,90 \\
\hline$+\mathbf{3 , 2 5}$ & 3,01 & 2,86 & 2,72 & 2,59 & 2,47 & 3,35 & 3,55 & 3,76 & 3,99 & 4,24 \\
\hline$+\mathbf{3 , 5}$ & 3,25 & 3,09 & 2,94 & 2,80 & 2,67 & 3,62 & 3,83 & 4,06 & 4,31 & 4,58 \\
\hline$+\mathbf{3 , 7 5}$ & 3,50 & 3,32 & 3,16 & 3,01 & 2,86 & 3,90 & 4,12 & 4,37 & 4,64 & 4,93 \\
\hline$+\mathbf{4}$ & 3,74 & 3,55 & 3,38 & 3,22 & 3,07 & 4,17 & 4,41 & 4,68 & 4,96 & 5,28 \\
\hline
\end{tabular}

According to the values shown in the tables above, the value of the lenses added, do not correspond exactly with the change in accommodation. As it can be seen in myope subjects when higher is the value of the $\mathrm{P}_{\mathrm{NL}}$ higher is the differences respect to the lens added. For example, a myopic subject of $-4 \mathrm{D}$ to which has added $+2 \mathrm{D}$, the theoretical variation of accommodation is $11^{\prime} 73 \mathrm{D}$, approximately 0 '25D lower. But if the myopic subject had $\mathrm{P}_{\mathrm{NL}}=-10 \mathrm{D}$, the difference would increase to $0.50 \mathrm{D}$ lower than the lens added. In these cases, since normal values have a standard deviation of 0 '50D, the values would be within the norm. Then a subject with a lens added of $+1^{\prime} 50 \mathrm{D}$ when $\mathrm{P}_{\mathrm{NL}}=-4 \mathrm{D}$, would be below the standard deviation since variation in accommodation really done would 1'29D, therefore the value would be correct. But in the example explained for $\mathrm{P}_{\mathrm{NL}}=-10 \mathrm{D}$, the value would be not correct.

In hyperopic refractions the measure of relative accommodation is underestimated. For instance, for a value of $\mathrm{P}_{\mathrm{NL}}=+6 \mathrm{D}$ and a lens added of $+2 \mathrm{D}$, the value of PRA would be 2 '27D. However, if the lens added is $+3.50 \mathrm{D}$, the theoretical accommodative change would be $4.06 \mathrm{D}$, being clinically relevant the difference. 
Table 4. NRA theoretical values based on the negative lenses added and the value of the myopic and hyperopic NL.

\begin{tabular}{|l|l|l|l|l|l|l|l|l|l|l|}
\cline { 2 - 13 } \multicolumn{1}{c|}{} & \multicolumn{9}{|c|}{$\mathbf{P}_{\mathbf{N L}}(\mathbf{D})$} \\
\hline $\mathbf{L}(\mathbf{- )}$ & $\mathbf{- 2}$ & $\mathbf{- 4}$ & $\mathbf{- 6}$ & $\mathbf{- 8}$ & $\mathbf{- 1 0}$ & $\mathbf{+ 2}$ & $\mathbf{+ 4}$ & $\mathbf{+ 6}$ & $\mathbf{+ 8}$ & $\mathbf{+ 1 0}$ \\
\hline $\mathbf{- 0 , 2 5}$ & 0,22 & 0,21 & 0,20 & 0,19 & 0,18 & 0,25 & 0,26 & 0,27 & 0,29 & 0,31 \\
\hline $\mathbf{- 0 , 5}$ & 0,44 & 0,42 & 0,40 & 0,38 & 0,36 & 0,49 & 0,52 & 0,55 & 0,58 & 0,62 \\
\hline $\mathbf{- 0 , 7 5}$ & 0,66 & 0,63 & 0,60 & 0,57 & 0,54 & 0,73 & 0,77 & 0,82 & 0,87 & 0,92 \\
\hline $\mathbf{- 1}$ & 0,88 & 0,83 & 0,79 & 0,76 & 0,72 & 0,97 & 1,03 & 1,09 & 1,15 & 1,22 \\
\hline $\mathbf{- 1 , 2 5}$ & 1,09 & 1,04 & 0,99 & 0,94 & 0,90 & 1,21 & 1,28 & 1,35 & 1,43 & 1,52 \\
\hline $\mathbf{- 1 , 5}$ & 1,31 & 1,24 & 1,18 & 1,13 & 1,08 & 1,45 & 1,51 & 1,62 & 1,71 & 1,82 \\
\hline $\mathbf{- 1 , 7 5}$ & 1,52 & 1,45 & 1,38 & 1,31 & 1,25 & 1,69 & 1,78 & 1,88 & 1,99 & 2,11 \\
\hline $\mathbf{- 2}$ & 1,73 & 1,65 & 1,57 & 1,50 & 1,43 & 1,92 & 2,03 & 2,14 & 2,27 & 2,41 \\
\hline $\mathbf{- 2 , 2 5}$ & 1,94 & 1,85 & 1,76 & 1,68 & 1,60 & 2,15 & 2,27 & 2,40 & 2,54 & 2,70 \\
\hline $\mathbf{- 2 , 5}$ & 2,15 & 2,05 & 1,95 & 1,86 & 1,78 & 2,39 & 2,52 & 2,66 & 2,82 & 2,99 \\
\hline $\mathbf{- 2 , 7 5}$ & 2,36 & 2,24 & 2,14 & 2,04 & 1,95 & 2,62 & 2,76 & 2,92 & 3,09 & 3,27 \\
\hline $\mathbf{- 3}$ & 2,57 & 2,44 & 2,33 & 2,22 & 2,12 & 2,84 & 3,00 & 3,17 & 3,36 & 3,56 \\
\hline $\mathbf{- 3 , 2 5}$ & 2,77 & 2,64 & 2,51 & 2,40 & 2,29 & 3,07 & 3,24 & 3,42 & 3,62 & 3,84 \\
\hline $\mathbf{- 3 , 5 0}$ & 2,97 & 2,83 & 2,70 & 2,57 & 2,46 & 3,30 & 3,48 & 3,66 & 3,89 & 4,12 \\
\hline $\mathbf{- 3 , 7 5}$ & 3,18 & 3,02 & 2,88 & 2,75 & 2,63 & 3,52 & 3,71 & 3,92 & 4,15 & 4,40 \\
\hline $\mathbf{- 4}$ & 3,38 & 3,22 & 3,07 & 2,92 & 2,79 & 3,74 & 3,95 & 4,17 & 4,41 & 4,68 \\
\hline
\end{tabular}

For myopic subjects, higher is the $\mathrm{P}_{\mathrm{NL}}$ value higher is the difference in the PRA calculated. For example, in a myopic subject with $\mathrm{P}_{\mathrm{NL}}=-4 \mathrm{D}$ to which have added $-2 \mathrm{D}$ there would be an accommodating variation of 1'65D, 0'35D lower than the value of the lenses added. If the $\mathrm{P}_{\mathrm{NL}}=-10 \mathrm{D}$ and the lens added is $-2 \mathrm{D}$ the theoretical value of the PRA should be $1^{\prime} 08 \mathrm{D}$, approximately $0.5 \mathrm{D}$ lower than the value that is considered normal in this measure.

In hyperopic subjects, depending on the value of $\mathrm{P}_{\mathrm{NL}}$, PRA measurement will be overestimated or underestimated. For $\mathrm{NL}$ higher than $+4 \mathrm{D}$ the theoretical accommodative variation is higher than the added lenses.

Thus, both for measurement of NRA as the PRA, the value of the lenses added do not exactly match the accommodative variation of the eye. The difference between the theoretical value and the lens added increases the value of the $\mathrm{P}_{\mathrm{NL}}$ and the dioptric value of the added lenses increases.

\section{CONCLUSIONS}

Measuring of the relative amplitude depends among other factors on accommodative stimulus value. The most of the peer-viewed literature establish a rule a test distance of $40 \mathrm{~cm}$ measured from the glasses $(2150 \mathrm{D}$ accommodative stimulus) in normal subjects.

In ametropic subjects we proved that, with the neutralizing lens, the accommodative stimulus does not match to 2'50D. This is because the eye is not accommodating in the position of the test, but in the test image through the lens. Therefore, the conditions under the test is performed are not the same for emmetropic and ametropic subjects. Therefore, the standard rule used is not valid for all cases.

Moreover, the distance of $40 \mathrm{~cm}$ is usually taken as reference for the measure of RNA and, however, the dioptric value of these lenses does not match the changes made by the eye in accommodation.

To determine the exact value of relaxing accommodation (RNA) and stimulating accomodation (PRA) a more precise calculation has to carry out. Depending on $\mathrm{P}_{\mathrm{NL}}$ and positive or negative lenses added the value of relative accommodation changes. Consequently not all subjects who accept the same value of lens added perform the same change in accommodation and therefore the NRA or PRA values would be different. 
We have found errors in the measure of the relative amplitudes clinically relevant, and can produce values that are not considered normal. Therefore, in these cases, the clinical conclusions would not be correct.

In order to optimize the measure, it is important that both ammetropic and emmetropic subjects perform the measurement under the same conditions. Therefore, we suggest that it would be desirable to place the test at the position of effective binocular object. Thus, in all cases, the eye will converge to $40 \mathrm{~cm}$ and accommodate $2.50 \mathrm{D}$ independently

the value of $\mathrm{P}_{\mathrm{NL}}$. Furthermore, from the value of the added lenses and the $\mathrm{P}_{\mathrm{NL}}$, it should calculate the change in accommodation to obtain more accurate values.

\section{REFERENCES}

[1] Rabbetts RB. “Cilincal visual optics”, Oxford, England: Butterworth- Heinemann, 243 (2000)

[2] Borish IM. "Clinical Refraction.Professional Press", Chicago, $3^{\text {rd }}$ ed, 764 (1970)

[3] Tunnancliffe A. "Introduction to Visual optics", Hurlingham Business Park, $4^{\text {th }}$ Edition, 323(1993)

[4] Sheiman M, Wick B. "Clinical Management of binocular vision”, Lippincott Williams \& Wilkins 464-465

[5] Zadnik, The ocular examination. W.B. Saunders Company. 1997:105-107.

[6] Morgan MW. "The clinical aspects of accommodation and convergence”, Am J Opt Physiol Opt, 21:301-313 (1944)

[7] Le Grand Y, “Optique physiologique: La dioptrique de lóeil et sa correction” Tomo I. Paris, Editions de la revue d'optique, 147-171 (1952)

[8] Pons A, Martinez F. "Fundamentos de la visión binocular". Publicaciones de la Universidad de Alicante, 152 (2004) 